livraisons

d'Histoire

de l'Architecture

\section{Livraisons de l'histoire de l'architecture}

$36 \mid 2018$

Jean-Louis Pascal (2)

\title{
Le concours pour la construction du Sacré-Cœur de Montmartre, une désillusion
}

The competition for the construction of the Sacre-Cour in Montmartre, a disillusionment

Der Wettbewerb zum Bau der Kirche Sacré-Cœur de Montmartre - Pascals Desillusionierung

\section{Anne Richard-Bazire}

\section{OpenEdition}

Journals

Édition électronique

URL : http://journals.openedition.org//ha/1021

DOl : $10.4000 /$ /ha. 1021

ISSN : 1960-5994

\section{Éditeur}

Association Livraisons d'histoire de l'architecture - LHA

Édition imprimée

Date de publication : 15 décembre 2018

Pagination : $31-49$

ISSN : 1627-4970

Référence électronique

Anne Richard-Bazire, « Le concours pour la construction du Sacré-Cœur de Montmartre, une désillusion », Livraisons de l'histoire de l'architecture [En ligne], 36 | 2018, mis en ligne le 15 décembre 2020, consulté le 24 janvier 2021. URL : http://journals.openedition.org//ha/1021 ; DOI : https:// doi.org/10.4000/lha.1021 


\section{LE CONCOURS POUR LA CONSTRUCTION DU SACRÉ-CCEUR DE MONTMARTRE, UNE DÉSILLUSION}

Le début des années soixante-dix est une période intense pour l'architecte Jean-Louis Pascal. En 1872, il prend la tête de l'atelier de Charles-Auguste Questel à l'École des Beaux-Arts, atelier dont il avait été l'élève dix-sept ans auparavant. Il participe également au concours pour la reconstruction de l'Hôtel de ville de Paris incendié lors des évènements de la Commune, dont il est éliminé au $3^{\mathrm{e}}$ tour de scrutin. Deux ans plus tard, en 1874, il participe au concours pour la construction, sur la colline de Montmartre, de la basilique du vœu national au Sacré-Cœur de Jésus. Il est nommé un an plus tard architecte de la Bibliothèque nationale de la rue de Richelieu, en remplacement d'Henri Labrouste subitement décédé, et architecte diocésain en charge du diocèse de Valence. Pascal mène toutes ces activités de front, travailleur infatigable ne s'autorisant que peu de loisirs ${ }^{1}$. En janvier 1874, une commission artistique de douze membres ${ }^{2}$ définit les conditions et le programme du concours public, ouvert du $1^{\text {er }}$ février au 30 juin 1874, destiné à choisir le maître d'œuvre du futur Sacré-Cœur de Montmartre ${ }^{3}$, le cardinal-archevêque Hippolyte Guibert se réservant toutefois la décision finale. Dans ce concours, au programme ${ }^{4}$ relativement précis, certaines exigences allaient conditionner la question du style. Il fallait remplir les conditions spéciales d'une église votive et de pèlerinage.

1. «Il habitait dans ce quartier bruyant du boulevard Saint-Denis, une vieille maison qui, par je ne sais quel miracle, était pleine de silence et de recueillement. Quand, de sa retraite, il descendait dans la rue, un étonnement quotidien l'arrêtait devant les cafés toujours pleins : "Que peut-on dire, que peut-on faire là-dedans ?” se demandait-il anxieux ». Charles-Marie Widor, Notice sur la vie et les æeuvres de Jean-Louis Pascal, 4 juin 1837 - 16 mai 1920, Institut de France, Académie des BeauxArts, 4 décembre 1920, Paris, Firmin-Didot, p. 14.

2. L'Archevêque désigna deux membres du Comité de l'œuvre pour faire partie de la commission: M. Cornudet, président du Comité, qui, malade, fut remplacé par M. Féline Romany et Alexandre Legentil, secrétaire du même Comité. Dix autres membres furent nommés, au sein desquels il y avait cinq architectes : Albert Lenoir, Théodore Ballu, Louis Duc, Henri Labrouste et Charles Rohault de Fleury et MM. Alphand, directeur des travaux de la ville de Paris, de Cardaillac, directeur des Bâtiments civils, Chesnelong, député, le baron de Guilhermy, conseiller à la Cour des comptes, Eugène Guillaume, directeur de l'École des Beaux-Arts ; s'y ajoutaient six jurés choisis par l'ensemble des candidats après la remise des projets : Émile Vaudremer, Charles Garnier, Léon Ginain, Charles Questel, Ernest Coquart et Hector Lefuel, donc quatre "proches» de Pascal, sur six.

3. Claude Laroche, "Le concours de 1874, ou le roman obligé ", Le Sacré-Cour de Montmartre. Un Vou National, sous la direction de Jacques Benoist, Délégation à l'Action Artistique de la Ville de Paris, 1995, p. 105-121.

4. "Programme d'un concours pour la construction de l'église du Sacré-Cour de Montmartre ", Le Moniteur des architectes, $n^{\circ}$ 8, 1874, p. 38-42. Souvenir du Concours de l'église du Sacré-Couur juillet 1874, 23 p., (« Rapport sur le concours de l'église du Sacré-Cœur », p. 3-10, et Louis Duc, «Rapport sur le jugement du concours de l'église du Sacré-Cour », p. 11-23). 


\section{La question du plan}

La configuration du terrain sur lequel devait s'élever le Sacré-Cœur de Montmartre et son orientation, étaient fixées ${ }^{5}$ : le périmètre, irrégulier dans ses faces latérales, était d'environ cent cinquante mètres de largeur sur trois cent dix mètres de longueur; l'axe longitudinal, allant du midi au nord, s'élevait avec une différence de niveau de cinquante mètres ${ }^{6}$. L'espace dévolu à l'église se présentait vers Paris avec une façade droite de cinquante mètres et se prolongeait sur une longueur de quatre-vingt-dix mètres, pour se terminer au chevet par un demi-cercle ${ }^{7}$. Cette disposition, rebelle à la croix latine, se prêtait plus facilement au plan centré. " $\mathrm{Ce}$ qui forçait presque à faire une architecture classique, c'est-à-dire païenne " ${ }^{8}$. Les dépendances occupaient la partie est du plateau. La construction des sacristies ${ }^{9}$, presbytère $^{10}$ et bâtiments de service sur un terrain distinct, pour conserver l'aspect d'isolement de l'église, impliquait leur réunion à l'église par un passage couvert ${ }^{11}$. Des "parties hautes, dômes, tours ou clochers" étaient imposées ainsi qu'une statue monumentale du Sacré-Cour placée extérieurement, "d'une manière trèsapparente ${ }^{12}$. Les concurrents devant proportionner leurs projets de construction à la solidité du terrain, une coupe géologique leur fut fournie en marge du plan ${ }^{13}$. Dans des propos extrêmement polémiques sur le style de l'édifice à construire, l'abbé Carle, dans la Semaine Religieuse de Nîmes, accusa les rédacteurs du concours d'avoir choisi à dessein l'orientation nord-sud de l'église pour en déterminer le plan, vu l'étroitesse du terrain dans ce sens, et donc le style :

"Le nord est le côté du mal, et la franc-maçonnerie a pour doctrine secrète, par dérision pour le Christ qui est le Bien, d'user de toute son influence pour faire tourner vers le nord les églises qui se construisent de nos jours [...] la commission artistique du vœu national avait parfaitement la liberté

5. Une sous-commission (Alphand, Ballu, Cardaillac, Duc et Rohault de Fleury) avait été constituée pour rédiger un avant-programme.

6. Claudius Lavergne, "Concours pour la construction de l'église du Sacré-Cœur à Montmartre ", L'Univers, $\mathrm{n}^{\circ} 2512$, dimanche 19 juillet 1874, p. 1-2.

7. "Programme d'un concours pour la construction de l'église du Sacré-Cour de Montmartre ", Le Moniteur des architectes, op. cit., article 5, p. 38.

8. L'église du Sacré-Coeur à Montmartre sera-t-elle de notre style national ou sera-t-elle d'un style étranger? par un comité d'archéologues, Paris, Féchoz, 1875, p. 12, extrait de la Décentralisation, 14 juin 1875.

9. Il y avait six sacristies distinctes : celle des messes pour les prêtres de l'église, celle des messes pour les prêtres de l'extérieur, une sacristie pour le matériel, une pour le service du pèlerinage, une pour les archives, une pour les chantres et un logement pour un sacristain-concierge. "Programme d'un concours pour la construction de l'église du Sacré-Cœur de Montmartre ", Le Moniteur des architectes, op. cit., article 14, p. 39.

10. Le presbytère devait comporter un cloître, un petit oratoire, une bibliothèque, une ou deux salles de réunion, deux salles-à-manger, une cuisine et ses dépendances et une cave. Ibid.

11. Ibid., article 13 , p. 39.

12. Ibid., articles 7 et 8 , p. 38 .

13. "Le plan sus-indiqué comprendra une coupe géologique et des profils de la partie de la colline de Montmartre sur laquelle devront être élevées l'église et ses dépendances ». Ibid., article 3, p. 38. 
du choix ; mais elle a voulu établir la façade en regard de Paris, et par suite, vu la prompte dépression du terrain au-delà du point fixé pour l'abside, se trouver dans la nécessité de ne donner au monument qu'une longueur à peine double de la largeur, ces proportions convenant particulièrement à un édifice à dômes " ${ }^{14}$.

Pour la solidité de l'édifice, le style ogival était aussi prôné :

«La meilleure économie était dans le choix du style ogival qui répartit le poids réel avec infiniment moins d'écrasement, grâce à l'emploi si ingénieux des forces agissantes et à la légèreté relative des matériaux [...] l'église bien orientée trouvait au couchant un terrain plus solide et surtout mieux appuyé, vu son éloignement des dépressions subites qui entourent, par trois côtés, l'emplacement si malencontreusement choisi ${ }^{15}$.

Soixante-dix-huit projets furent présentés ${ }^{16}$; quarante-sept émanaient de l'École des Beaux-Arts dont six de grands prix de Rome, dus à Louis François Philippe Boitte (1830-1906), Constant Moyaux, Louis Noguet, Jean-Louis Pascal, Albert Bénard et Charles Alfred Leclerc. Le jury procéda à trois tours d'éliminations successives, à l'issue desquels treize projets furent conservés ${ }^{17}$. Les trois premiers prix furent attribués à : Paul Abadie ${ }^{18}$ (1812-1884) (ill. 1 et 2), nommé au second tour du scrutin par douze voix, Gabriel Davioud et Charles Lameire (1832-1910), nommés au sixième tour par dix voix et Charles-Henri Cazaux (1845-1906), nommé au premier tour de scrutin par dix voix ${ }^{19}$. Les projets suivants reçurent des indemnités ${ }^{20}$. Jean-Louis Pascal (ill. 3 et 4 ) arriva dixième, nommé au troisième tour de scrutin par onze voix.

14. L'église du Sacré-Coeur à Montmartre sera-t-elle de notre style national ou sera-t-elle d'un style étranger? par un comité d'archéologues, Paris, Féchoz, 1875, p. 39-40, extrait de la Semaine Religieuse de Nîmes, 20 juin 1875.

15. Ibid., p. 46. Les fondations de l'église se composent de quatre-vingt-trois puits de $33 \mathrm{~m}$ de profondeur sur 4,50 $\mathrm{m}$ de diamètre en moyenne, remplis de maçonnerie très solide; ils sont reliés entre eux par des arcs en pierre de taille. Basilique du Sacré-Cour de Jésus à Montmartre, Paris, imp. F. Levé, s.d., $71 \mathrm{p}$.

16. Chaque projet devait présenter : un plan de la crypte, de l'église, des tribunes, des combles; une élévation de la façade principale et d'une façade latérale; des coupes longitudinales et transversales de l'église; des plans de la sacristie et du presbytère. Les plans, coupes et élévations devaient être à l'échelle de $1 \mathrm{~cm} / \mathrm{m}$; on devait y joindre un plan d'ensemble de l'église, de ses dépendances, de ses abords et de la promenade projetée à l'échelle de $4 \mathrm{~mm} / \mathrm{m}$.

17. Les projets de MM. Roux, Pascal, Moyaux, Leclerc, Douillard frères (2 projets), Davioud et Lameire, Coisel, Chipiez, Cazaux, Bernard et Tournade, Abadie, Raulin et Dillon. Quatre autres projets furent rajoutés par la suite : ceux de MM. Letz, Magne, Métivier et Rouyer, des mentions honorables devant être attribuées aux cinq projets classés après le dixième.

18. Claude Laroche (dir.), Paul Abadie, architecte. 1812-1884, Paris, éd. de la R.M.N., 1988, 372 p.

19. Ils recevaient respectivement : 12000,8000 , et $5000 \mathrm{fr}$.

20. MM. Douillard étaient classés quatrièmes, Bernard et Tournade : cinquièmes, Coisel : sixième, Moyaux : septième, Roux : huitième et Raulin et Dillon : neuvièmes. Les sept projets primés recevaient $1500 \mathrm{fr}$. 


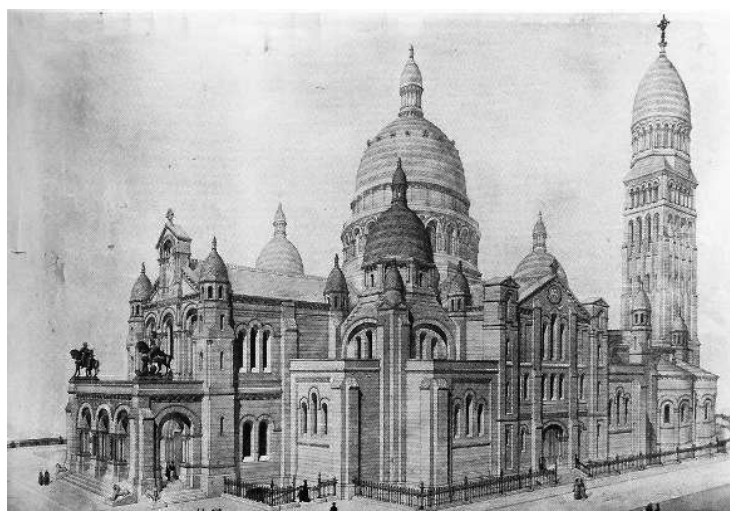

Ill. 1 : Projet lauréat du concours pour la construction du Sacré-Coeur de Montmartre. Paul Abadie. Vue perspective. 1874. Claude Laroche (dir.), Paul Abadie, architecte. 1812-1884, Paris, éd. de la R.M.N., 1988, p. 229.

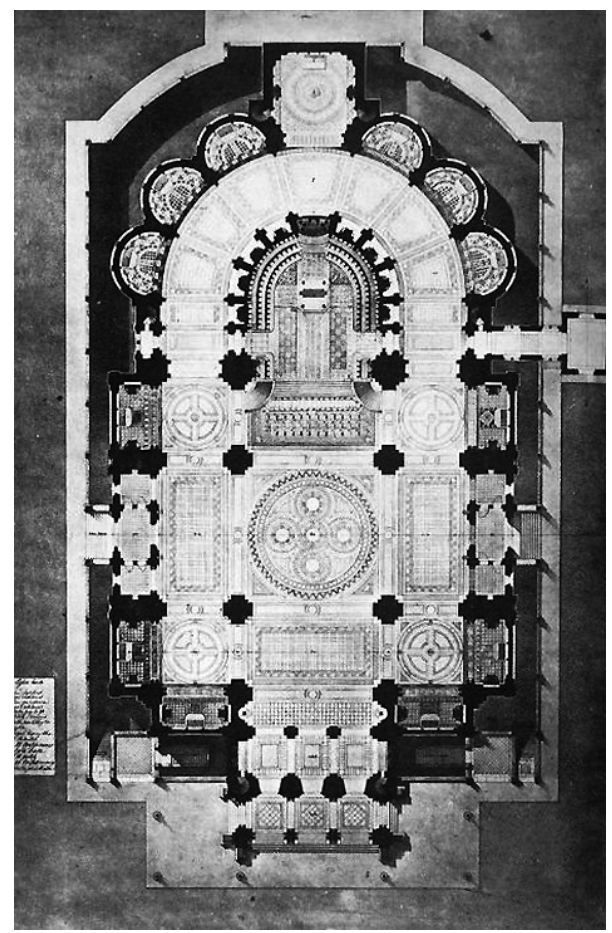

Ill. 2 : Projet lauréat du concours pour la construction du Sacré-Cour de Montmartre. Paul Abadie. Plan. 1874. Claude Laroche (dir.), Paul Abadie, architecte. 1812-1884, Paris, éd. de la R.M.N., 1988, p. 230 . 


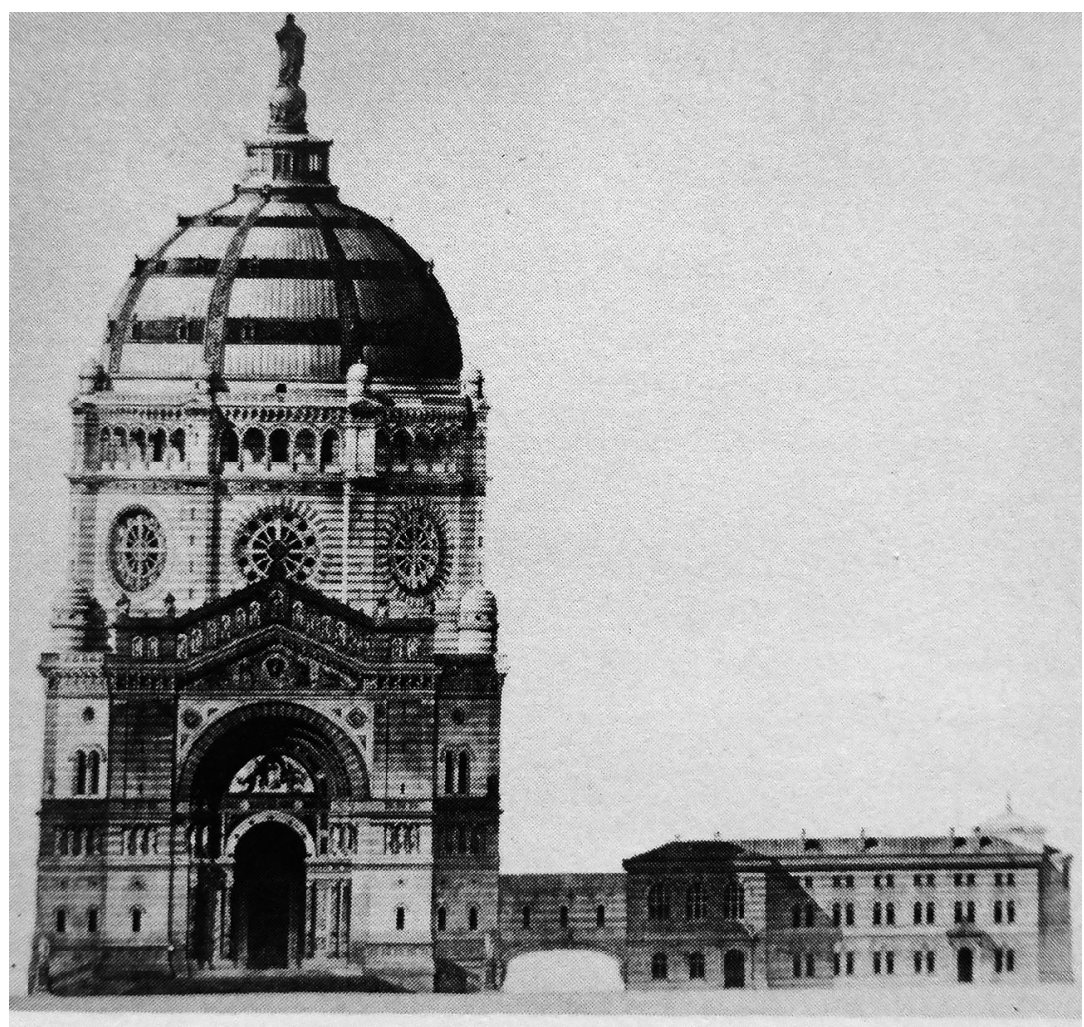

Ill. 3 : Projet du concours pour la construction du Sacré-Cour de Montmartre. Jean-Louis Pascal. Élévation. 1874. Claude Laroche (dir.), Paul Abadie, architecte. 1812-1884, Paris, éd. de la R.M.N., 1988 , p. 219.

«MM. Pascal, Bernard et Tournade, Roux et Chipiez (ill. 4), ont adopté un parti complètement différent de ceux des autres concurrents. Ils ont adopté un plan circulaire et ont donné une grande importance à la coupole ${ }^{21}$.

C’est ce qui faisait la beauté du projet de Pascal, mais qui fit aussi sa perte. Tous les critiques louèrent la magnificence du dôme de Pascal :

"J'avoue cependant que pour embrasser, circonvenir et soulever le dôme immense du projet de M. Pascal, je me sens moins fort. Pourtant ce projet doit compter parmi les plus beaux et ce qu'il offre de ressemblance avec le dôme de Santa Maria del Fiore, de Brunelleschi, n'est pas pour nous en éloigner $»^{22}$.

21. "Programme d'un concours pour la construction de l'église du Sacré-Cour de Montmartre ", Le Moniteur des architectes, $\mathrm{n}^{\circ} 1874$, p. 113-120.

22. Claudius Lavergne, "Concours pour la construction de l'église du Sacré-Cour à Montmartre ", L'Univers, $\mathrm{n}^{\circ} 2518$, samedi 25 juillet 1874, p. 1-2. 


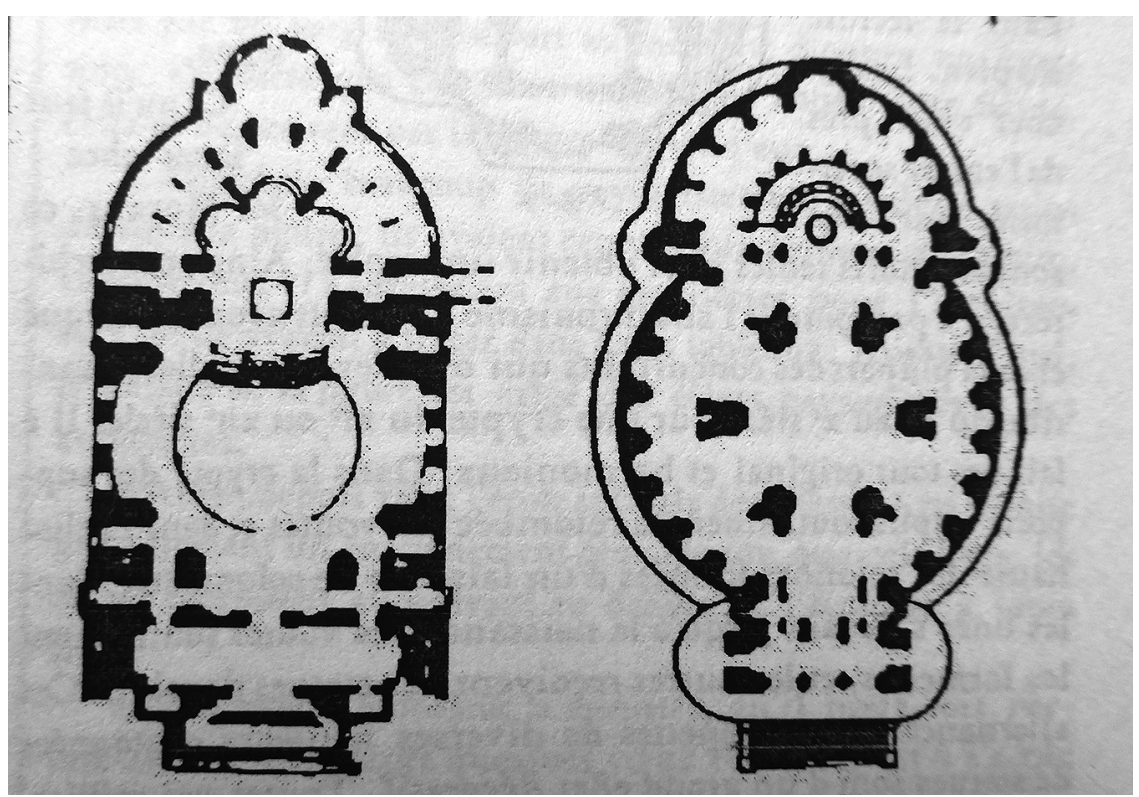

Ill. 4 : Projets du concours pour la construction du Sacré-Cour de Montmartre. Jean-Louis Pascal (gauche) et Charles Chipiez. Plans. 1874. Moniteur des Architectes, 1874, p. 118.

C'était aussi l'opinion de l'architecte L. Cernesson, qui écrivit dans la R.G.A.T.P. :

«M. Pascal s'est préoccupé visiblement du site choisi en établissant un dôme en quelque sorte colossal dont l'effet est grandiose. La carrure des pendentifs à la base répond aux dimensions de la coupole; les quatre arcs-doubleaux sont des voûtes cylindriques d'une grande largeur, dont l'une abrite le maître-autel, pour lequel on pourrait peut-être désirer une place plus noble. Quoi qu'il en soit, le plan qui en résulte présente de l'unité : il permet une circulation facile et tout l'ensemble est plein de majesté et de grandeur. » ${ }^{23}$

Jean-Louis Pascal s'était inspiré du plan du Saint-Sépulcre à Jérusalem. La coupe du vaste dôme (ill. 5) inspiré par la Renaissance italienne faisait un grand effet ; sa sacristie était jugée "très jolie » et sa terrasse "très bien réussie pour les piétons " ${ }^{24}$. Paul Abadie, Charles-Henri Cazaux (1845-1906) et Louis François Roux (1838-1921) avaient opté pour une nef de plan centré carré avec un chœur développé et un large déambulatoire sur lequel s'ouvraient les chapelles rayonnantes. Le plan centré

23. Louis Cernesson, "Concours pour la construction d'une église du Sacré-Cour à Montmartre ", R.G.A.T.P., t. XXXI, 1874, p. 181-186, p. 184.

24. "Concours d'architecture pour la construction de l'église du Sacré-Cœur, à Paris-Montmartre ", Le Bâtiment, journal des travaux publics et particuliers, $\mathrm{n}^{\circ} 50$, dimanche 26 juillet 1874, p. 1-2. 


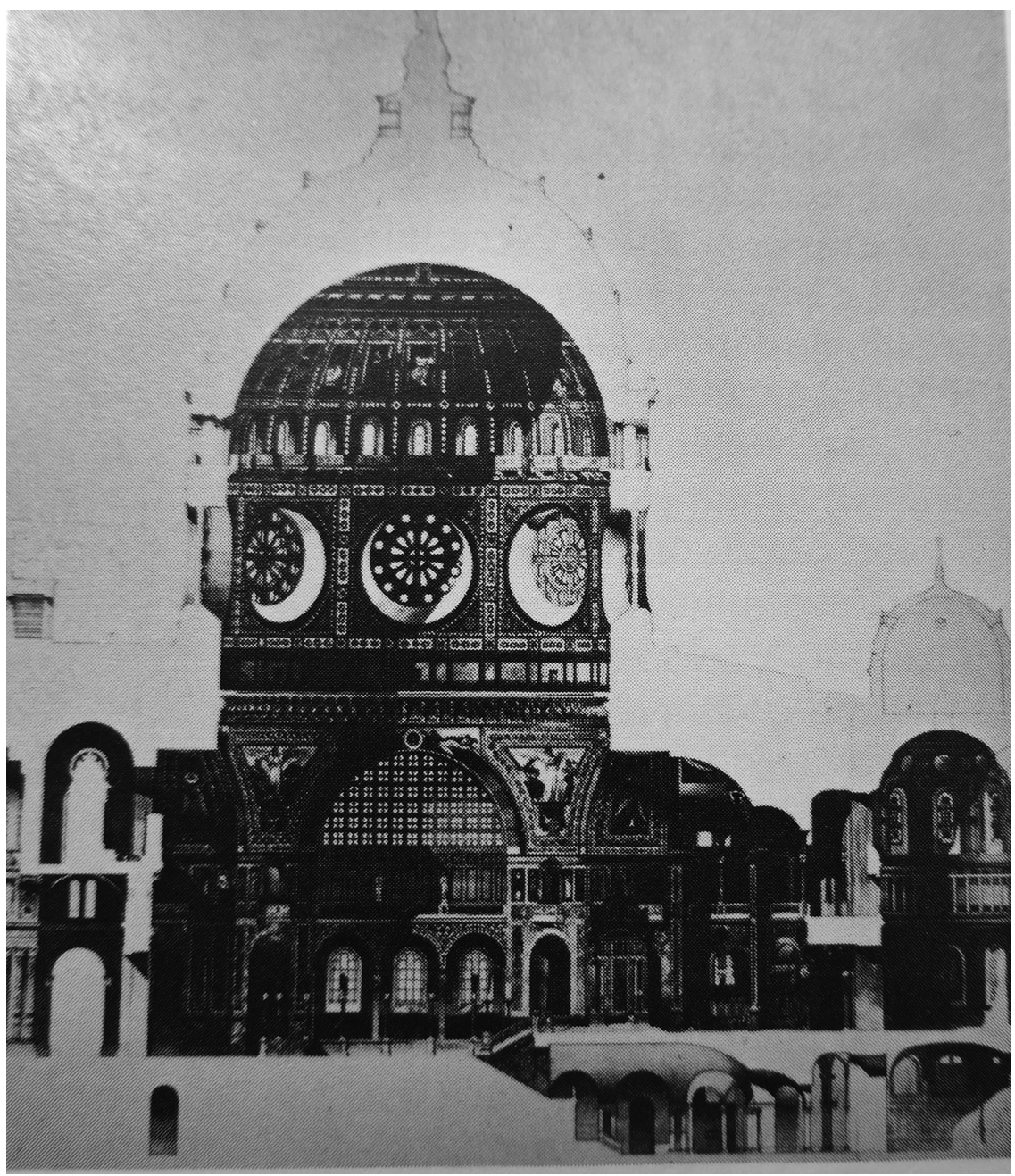

Ill. 5: Projet du concours pour la construction du Sacré-Cour de Montmartre. Jean-Louis Pascal. Coupe longitudinale. 1874. Claude Laroche (dir.), Paul Abadie, architecte. 1812-1884, Paris, éd. de la R.M.N., 1988, p. 219.

et le chœur développé permettaient un auditoire groupé. C’était aussi le plan des martyria, ce qui convenait pour un "Mont des martyrs » ${ }^{25}$. Jean-Louis Pascal, F. Bernard et Albert Marie Tournade (1847-1891), mais aussi d'autres concurrents : Gabriel Davioud et Charles Lameire, Constant Moyaux, Jérôme Charles Chipiez

25. Claude Laroche, "Le concours de 1874 ", Paul Abadie, architecte. 1812-1894, op. cit., p. 211-227, note $\mathrm{n}^{\circ} 22$, p. 118 : intervention de M. J.-M. Leniaud lors de la soutenance de thèse de J. Benoist, le 13 avril 1991 à la Sorbonne. 
(1835-1901), René Deminuid (1835-1881) et Désiré Henri Louis Devrez (18241896), choisirent de mettre en avant le caractère votif de l'édifice marqué par la coupole proéminente abritant généralement le sanctuaire, mais déterminant autour d'elle un espace parfois réduit à sa plus simple expression : " Ils [Bernard et Tournade] ont pris corps à corps l'idée du culte du Sacré-Cour ; ils en ont fait l'âme de leur monument, et, rejetant toutes les dispositions accessoires pour mieux l'accentuer, l'édifice n'est plus qu'un vaste dôme et un vestibule d'introduction " ${ }^{26}$. Les projets des frères Louis François (1823-1896) et Lucien Michel Douillard (1829-1888), de Gustave Laurent Raulin (1837-1910) et Albert Nicolas Dillon (1843-1878), d'Auguste Joseph et Lucien Magne (1849-1916), de Claude Alexandre Nicolas Sandier et d'Alfred Isidore Coisel (1839-1883), avaient adopté un plan en forme de croix latine - avec autel «à la romaine » chez Coisel, et chez Raulin et Dillon ce qui gênait l'organisation des processions et diminuait l'impact des prédications dans une église qui était d'abord une église de pèlerinage.

\section{Une église de pèlerinage}

Pour organiser ces processions, le programme du concours demandait que les concurrents présentent un projet des rampes d'accès et de la promenade à créer sur les espaces destinés à cet effet ${ }^{27}$. Il était précisé que l'on pouvait élever en avant de la façade un perron ou un porche ${ }^{28}$. Une crypte communiquant avec l'église supérieure devait être construite sous le chœur et les bas-côtés et avoir des accès extérieurs ${ }^{29}$. Les bas-côtés devaient tourner autour du sanctuaire ${ }^{30}$ et être surmontés de galeries ou tribunes ${ }^{31}$. On souhaitait aussi un chœur ample et au moins vingt chapelles $^{32}$, dont une spécialement dédiée à la Vierge ${ }^{33}$. Il fallait «qu'une sainte table, aussi étendue que possible et d'un accès facile, favorise les communions ; que, près de l'autel, une place convenable soit réservée pour le cas où un prélat officierait pontificalement ${ }^{34}$.

26. Joseph Louis Duc, "Rapport sur le jugement du concours de l'église du Sacré-Cœur ", Rapport sur le concours de l'église du Sacré-Cour, Paris, Jules Le Clère et C $C^{\mathrm{ie}}, 1874$, p. 13.

27. "Programme d'un concours pour la construction de l'église du Sacré-Cœur de Montmartre ", Le Moniteur des architectes, op. cit., article 4, p. 38.

28. Ayant au maximum $25 \mathrm{~m}$ de largeur sur $10 \mathrm{~m}$ de profondeur. Ibid., article 5, p. 38.

29. "Programme d'un concours pour la construction de l'église du Sacré-Cœur de Montmartre ", Le Moniteur des architectes, op. cit., article 6, p. 38.

30. Pour faciliter les processions inhérentes à une église de pèlerinage. Ibid., article 9, p. 39.

31. Il fallait assurer aux prédications un large auditoire. Ibid., article 9, p. 39.

32. Cela permettait aux nombreux prêtres pèlerins de dire des messes en même temps.

33. "Programme d'un concours pour la construction de l'église du Sacré-Cœur de Montmartre ", Le Moniteur des architectes, op. cit., article 11, p. 39.

34. Souvenir du Concours de l'église du Sacré-Cœur, juillet 1874, p. 5 ("Rapport sur le concours de l'église du Sacré-Cœur ", p. 3-10, et Louis Duc, "Rapport sur le jugement du concours de l'église du Sacré-Cour ", p. 11-23). 
L'échec de Jean-Louis Pascal au concours vint du fait que son projet répondait complètement à la demande d'un monument votif et oubliait celle d'une église de pèlerinage :

«Ce projet s'est fait remarquer par sa physionomie particulière. Le caractère en est ferme et monumental. La pensée de son auteur a été de produire un grand effet par l'unité et la simplicité de la composition. Aussi, pour obtenir ce résultat, a-t-il sacrifié plusieurs parties accessoires mais essentielles. L'idée dominante de la composition est une vaste coupole assise sur un quadrilatère formé de robustes constructions. Mais ce projet est incomplet du point de vue du programme. Il n'y a ni nef, ni bas-côtés, ni chapelles proprement dites. La circulation, désirable pour le parcours des processions est indéterminée; sur quelques points même, elle serait impossible » 35 .

Pascal sacrifia - peut-être un peu trop - au «sacro-saint principe unitaire de la composition "Beaux-Arts", principe auquel sacrifièrent la plupart des concurrents, concentrés sur l'exaltation de leur seul dôme ", en réalisant un projet " unitaire jusqu'à l'extrême » ${ }^{36}$. Charles Lucas, dans des lettres adressées à l'abbé Corblet, considérait même que le projet de Pascal n'était plus une église :

«Une puissante unité obtenue à l'aide d'un grand parti, auquel tout est sacrifié aussi bien en plan qu'en élévation, caractérise le projet de M. Pascal. Ce n'est plus une église, et certains services de l'église, demandés par le programme, se placent difficilement avec leurs données conventionnelles dans cet édifice qui semble conçu dans le but unique de faire d'un dôme unique et gigantesque un piédestal monumental à la statue du Sacré-Cœur » ${ }^{37}$.

Les grandes célébrations inhérentes à une église de pèlerinage nécessitaient un chœur assez vaste pour que l'office public et même l'office pontifical puissent être célébrés avec la pompe convenable ${ }^{38}$. C'est pourquoi le projet de Davioud et Lameire donnait au chœur une place prépondérante avec un plan influencé par l'église Sainte-Marie-de-la-Fleur à Florence (dont Pascal s'inspira pour les oculi géants de sa coupole ${ }^{39}$ ) ou Saint-Augustin à Paris dont Bernard et Tournade s'inspirèrent aussi pour le chœur de leur projet (ill. 6). Il y eut très peu de projets néogothiques ${ }^{40}$

35. Ibid., p. 18 .

36. Claude Laroche, "Anatomie d'une chimère. Genèse et fortune du projet d'Abadie ", Paul Abadie, architecte. 1812-1894, op. cit., p. 228-251.

37. Charles Lucas, Un projet de décoration du Panthéon et le concours de l'église du Sacré-Cour à Montmartre, lettres adressées à M. l'Abbé J. Corblet, Paris, éd. Palmé-Ducher, 1875, p. 35.

38. "Programme d'un concours pour la construction de l'église du Sacré-Cour de Montmartre ", Le Moniteur des architectes, op. cit., article 10, p. 39.

39. François Loyer, "Une basilique synthétique ", Paul Abadie, architecte. 1812-1894, op. cit., p. 190-199.

40. Anatole de Baudot fournit le seul projet des XII ${ }^{e}$ et XIII ${ }^{e}$ siècles, retenu parmi les vingt-six concurrents choisis en première instance, mais non retenu dans les treize projets retenus en deuxième instance. 


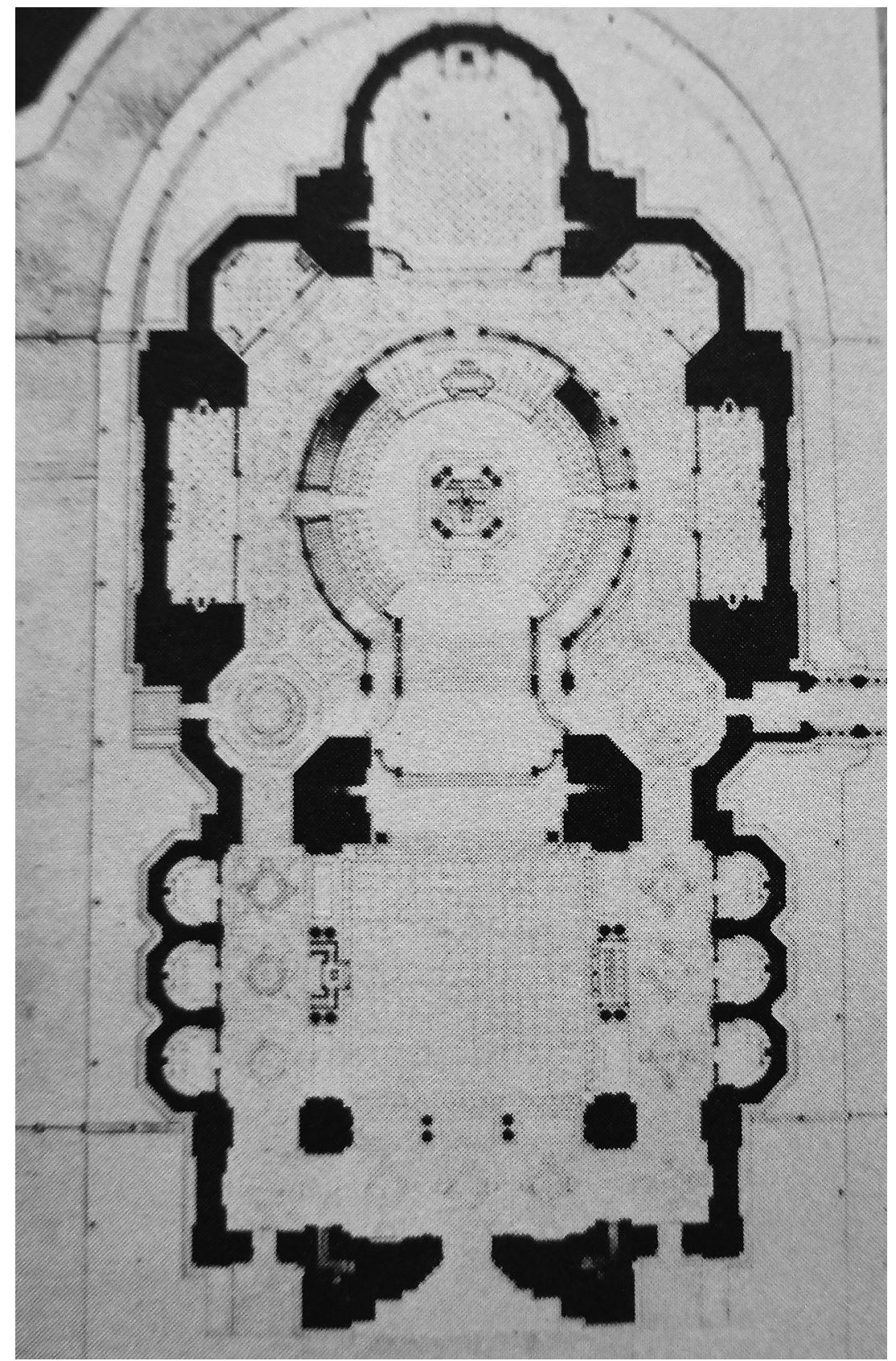

Ill. 6 : Projet du concours pour la construction du Sacré-Cœur de Montmartre. F. Bernard et A.-M. Tournade. Plan. 1874. Claude Laroche (dir.), Paul Abadie, architecte. 1812-1884, Paris, éd. de la R.M.N., 1988, p. 216. 




Ill. 7 : Projet du concours pour la construction du Sacré-Cour de Montmartre. F. Bernard et A.-M. Tournade. Élévation. 1874. Claude Laroche (dir.), Paul Abadie, architecte. 1812-1884, Paris, éd. de la R.M.N., 1988, p. 216.

à cause de la forme du terrain et à cause du programme qui s'opposait à celui d'une cathédrale; l'ordre classique était presque absent ${ }^{41}$. Le Moyen Âge italien,

41. Il y eut deux projets classiques : celui de Noguet d'une austère gravité : "Noguet a érigé des consoles en support exhaussant la tiare pontificale avec la triple couronne et la croix au sommet " et celui de Crépinet qui adopte au Sacré-Cœur le style de Mansart et réalise un dôme trop semblable à celui des Invalides. Claudius Lavergne, "Concours pour la construction de l'église du Sacré-Cœur à Montmartre ", L'Univers, no 2518, samedi 25 juillet 1874, p. 1-2. 
en revanche inspira les concurrents : Sienne et Orvieto pour la façade de Moyaux ; Pise pour le tambour de la coupole de Bernard et Tournade ${ }^{42}$ (ill. 7). Les projets devaient comprendre aussi la décoration et l'ameublement de l'église ${ }^{43}$. Ce fut autant l'adéquation au caractère exceptionnel du vœu que la réponse aux exigences formelles qui déterminèrent le jugement. Le rapport dira, sur le projet de Pascal : "On ne s'explique que difficilement la disposition du mobilier sous la coupole; on y trouve à regret une ressemblance fâcheuse avec l'aménagement d'un théâtre " ${ }^{4}$. Le jury reprochait également au projet de Davioud et Lameire d'avoir sacrifié l'architecture des bas-côtés : « de pauvres galeries latérales, basses, dont l'architecture est mesquine, [et] qui ne sont pas en rapport avec la majesté et la pompe des processions ", aux tribunes " qui, par leur disposition théâtrale, nuisent à l'effet religieux de l'édifice " ${ }^{45}$. À l'inverse, on reprochait au projet des frères Douillard, qui présentait "une belle et riche disposition en accord presque parfait avec les conditions du programme ", c'est-à-dire une église avec un grand chœur destiné à un culte spécial, ses façades latérales, "qui présentent un caractère froid et monastique qui va à l'encontre du sentiment votif de cette église " ${ }^{46}$. Les anciennes notions de convenance d'un projet à un programme et de caractère de l'édifice étaient toujours d'actualité ; le jury trouvait que les dispositions extérieures, pour l'accès au monument, de Davioud, étaient traitées dans un style " peu convenable au sujet " ${ }^{4}$, même si dans la presse on pouvait lire que ces squares, ces grottes, ces cascades et ces bassins aux eaux jaillissantes formaient au monument de l'église " une base vraiment belle, solide et grandiose ", caractérisée en un mot d' "ordonnance haussmannienne digne de la Babylone moderne ${ }^{48}$.

«Davioud n'a pu oublier qu'il était l'architecte des promenades de Paris ; il a vu dans les pénitentes au Sacré-Cœur de Jésus, les charmantes habituées des promenades du lac; il a songé à leurs pieds mignons et à leur teint de lys [...] Il a transformé le calvaire de Montmartre en un chemin de roses, et ce sybaritisme par trop $\mathrm{XIX}^{\mathrm{e}}$ siècle enlève au projet le caractère religieux, l'absorbe et lui nuit ${ }^{49}$.

Ailleurs, on lisait : "La disposition intérieure suivie par M. Pascal rappelle un peu trop celle des salles de spectacle; mais quelle franchise dans cette puissante

42. Claude Laroche, "Le concours de 1874 ", Paul Abadie, architecte. 1812-1894, op. cit., p. 220.

43. "Programme d'un concours pour la construction de l'église du Sacré-Cœur de Montmartre ", Le Moniteur des architectes, op. cit., article 16, p. 40.

44. Joseph Louis Duc, "Rapport sur le jugement du concours de l'église du Sacré-Cour ", Souvenir du Concours de l'église du Sacré-Cour, op. cit., p. 18.

45. Ibid., p. 14.

46. Ibid., p. 15.

47. Ibid., p. 14 .

48. "Concours, Vœu national du Sacré-Cour, compte-rendu de l'exposition ", la Gazette des architectes et du bâtiment, 1874, p. 115-120.

49. "Programme d'un concours pour la construction de l'église du Sacré-Cœur de Montmartre ", Le Moniteur des architectes, op. cit., p. 116-117. 
étude ! La façade, traitée dans le style lombard, a beaucoup de couleurs, et ce projet est incontestablement un des plus originaux et un des meilleurs " ${ }^{50}$. Louis Duc louait le projet de Pascal pour son « architecture monumentale » révélatrice du «talent sérieux de son auteur " 51 . Il faut surtout signaler dans cette œuvre remarquable le porche avec une seule arcade surmontée du Christ, des Apôtres et des Évangélistes, les huit oculi gigantesques et l'arcature placée à la naissance du dôme, enfin tout l'ensemble du presbytère ${ }^{52}$. Charles Garnier et Charles Rohault de Fleury soutenaient Pascal et Bernard, qui avaient fait de très beaux dômes, et dont les projets étaient d'ailleurs très proches ${ }^{53}$. Pascal remercia d'ailleurs Garnier d'avoir voté pour lui, dans une lettre datée du 29 juillet $1874^{54}$. Mais Garnier admirait le plan d'Abadie, et il reprochait à ses deux favoris de n'avoir pas pensé aux processions ${ }^{55}$.

«En arrivant devant le projet Abadie, on est d'abord frappé de la beauté de son plan ${ }^{56}$ et de l'étude qui a présidé à son tracé. La simplicité de ses lignes, le dôme savamment placé au centre, le rayonnement des chapelles chorales autour de la tribune, l'heureuse et savante disposition des collatéraux que les processions doivent suivre et auquel l'architecte a pris soin d'indiquer la voie, l'entrée directe dans la crypte, le grand nombre de fenêtres qui l'éclairent suffisent à faire de ce projet un des meilleurs de l'exposition " $\$ 7$.

Ce furent Charles Garnier, Rohault de Fleury père et Louis Duc qui enlevèrent le jury. Louis Duc était un homme charmant, "aimable, de la manière la plus opposée possible à la façon dont Charles Garnier était aimable et entraînant " 58 .

50. Ibid., p. 118 .

51. Joseph Louis Duc, "Rapport sur le jugement du concours de l'église du Sacré-Cour », Souvenir du Concours de l'église du Sacré-Cour, op. cit., p. 18.

52. Charles Lucas, Un projet de décoration du Panthéon et le concours de l'église du Sacré-Cour à Montmartre, op. cit., p. 35-36.

53. «MM. Bernard et Tournade se rapprochent beaucoup du projet précédent [celui de Pascal], tant par le plan que par le style employé ; mais ils ont donné une ampleur moins magistrale à leur dôme par l'emploi de nombreuses colonnettes qui le supportent ". "Programme d'un concours pour la construction de l'église du Sacré-Cœur de Montmartre ", Le Moniteur des architectes, op. cit., p. 118.

54. Bibliothèque de l'École des Beaux-arts (B.E.B.A.), Ms 744, Lettres de l'architecte Jean-Louis Pascal à Charles Garnier, lettre no 63, 29 juillet 1874.

55. Hubert Rohault de Fleury, Historique de la Basilique du Sacré-Cour, pièces et documents, Paris, Levé, 1903 , t. 1, p. 207.

56. "Le plan [d'Abadie] est le plan central, cruciforme, inscrit dans un carré, dont les angles sont couverts par des coupolettes qui aident à contrebuter la grande coupole médiane. C'est le vieux type qu'on trouve en Syrie à Rufasah dès le $\mathrm{VI}^{\mathrm{e}}$ siècle, qui passa en Arménie, en Europe, où il inspira Germigny-des-Prés au IX ${ }^{\mathrm{e}}$ siècle. Abadie a combiné ce plan avec celui de Saint-Marc de Venise et de Saint-Front de Périgueux, mais a donné à la grande coupole une échelle plus vaste, comme Bramante et Michel-Ange dans leurs projets pour Saint-Pierre de Rome ". Louis Hautecœur, Histoire de l'architecture classique en France, la fin de l'architecture classique (1848-1900), t. VII, Paris, Picard, 1957, p. 213-214.

57. Hubert Rohault de Fleury, Historique de la Basilique du Sacré-Coeur, pièces et documents, op. cit., p. 207-208.

58. Ibid., p. 207. 
Charles Rohault de Fleury était «la raison même ", avec des opinions " mathématiquement formulées ", selon son fils, alors que Charles Garnier "au contraire, ne raisonnait pas, il sentait ${ }^{59}$ ".

«Le système de M. Pascal représenté par plusieurs projets de grande valeur a été énergiquement soutenu par MM. Duc, Garnier et Rohault de Fleury père et si le plan de Pascal avait répondu au programme, il aurait eu beaucoup de chances d'avoir le prix $"{ }^{60}$.

Par la suite, Charles Garnier soutint le plan d'Abadie qui obtint le premier prix dès le second tour de scrutin, par douze voix, contre cinq à Bernard et Tournade, et une à Constant Moyaux ; "Ce fut un choix de prudence; ou plutôt, ce fut moins peut-être le projet qu'on choisit que l'homme. Il était alors dans toute sa maturité, connu par de beaux travaux d'architecture religieuse, expérimenté et sûr " ${ }^{61}$. C'est à l'art de Sainte-Sophie de Constantinople qu'Abadie avait demandé son inspiration.

\section{Une église de style national, mais lequel?}

Une nouvelle fois, la production architecturale du $\mathrm{XIX}^{\mathrm{e}}$ siècle allait être taxée de pastiche, pourtant, à la différence du concours pour la reconstruction de l'Hôtel de Ville, où la nécessité de conserver la façade ancienne rendait le style Renaissance obligatoire, le style n'était pas imposé pour cette basilique du "vœu national ». Et cependant, le jury trouva que le caractère de l'édifice proposé par Jean-Louis Pascal, " trop exactement emprunté aux monuments byzantins ", était " peu en accord avec celui qu'il fallait rechercher pour l'objet du programme. Cet édifice serait certainement mieux placé à Jérusalem qu'à Paris " ${ }^{62}$. Pour d'autres, le style adopté par Pascal rappelait à la fois les monuments de Florence et de Constantinople ${ }^{63}$. "L'épithète "byzantin" et même "romano-byzantin" 64 employée pour décrire bon nombre de projets n'était pas faite pour rasséréner les tenants du "style national" " ${ }^{65}$ en ces années

59. Ibid.

60. Hubert Rohault de Fleury (Archives de la basilique du Sacré-Cœur de Montmartre, recherches de N. van der Meer) cité dans Claude Laroche, "Le concours de 1874 ", Paul Abadie, architecte. 1812-1894, op. cit., note 68, p. 227.

61. Julien Guadet, "L'église du Sacré-Cœur ", La Revue de l'Art ancien et moderne, Paris, 1900, p. 103120.

62. Joseph Louis Duc, «Rapport sur le jugement du concours de l'église du Sacré-Cour », Souvenir du Concours de l'église du Sacré-Cour, op. cit., p. 18.

63. Charles Lucas, Un projet de décoration du Panthéon et le concours de l'église du Sacré-Caur à Montmartre, op. cit., p. 35.

64. "On nomma "romano-byzantines" les églises où se mêlèrent les souvenirs de Périgueux, d'Angoulême, des autres édifices à coupoles de l'Aquitaine et ceux des édifices orientaux ". Louis Hautecœur, Histoire de l'architecture classique en France, la fin de l'architecture classique (1848-1900), op. cit., p. 211.

65. Claude Laroche, "Le concours de 1874, ou le roman obligé ", Le Sacré-Coeur de Montmartre. Un vou National, op. cit., p. 115. 
d'exacerbation de la conscience nationale. Le projet de Bernard et Tournade, proche de celui de Pascal par l'emploi d'assises aux couleurs alternées, fut également mal jugé. "Le plan a la grandeur et la simplicité de certains monuments étrangers à nos âges et à nos mœurs, mais [...] les effets produits par les matériaux de couleur semblent avoir une importance lointaine et étrangère peu en accord avec notre style national " 66 .

Julien Guadet fit une critique assez acerbe de l'édifice de Paul Abadie dans La Revue de l'Art ancien et moderne:

"Fidèle à l'inspiration des églises du sud-ouest de la France, il [Abadie] en a pris les façades, souvent charmantes de détails, mais qui ne sont en somme qu'un mur à l'extrémité d'une nef [...] Mais ne cherchez pas là la hardiesse des piliers gothiques, l'affinement voulu de la matière qui caractérise surtout le $\mathrm{XIV}^{\mathrm{e}}$ et le $\mathrm{XV}^{\mathrm{e}}$ siècle ; au contraire, ici tout est trapu, d'apparence solide et plutôt grosse, d'aspect un peu lourd ${ }^{67}$.

Et pourtant, le style ogival paraissait inadapté à de nombreux critiques, car ses formes élégantes ne pouvaient être vues et comprises qu'à des distances médiocres et dans des lieux moins déprimés. En perspective, les flèches disparaissent sur les grandes hauteurs, dans l'air qui les absorbe ${ }^{68}$, comme l'explique un critique décrivant un projet "gothique " présenté au concours :

"Il est vraiment singulier de voir combien ici les arcs en tiers point, les voûtes d'arête, les tours, les flèches, les contreforts sont maigres, chancelants, agaçants et produisent peu d'effet [...] on dirait une forêt de peupliers tremblants de peur et mûrs pour la cognée " ${ }^{69}$.

D'autre part, la volonté d'avoir des tribunes au-dessus des bas-côtés proscrivait l'architecture ogivale qui a fait des tribunes antiques le triforium de nos cathédrales du XIII ${ }^{\mathrm{e}}$ siècle ${ }^{70}$. Julien Guadet reprochait surtout à Abadie d'avoir raté sa façade et de ne pas avoir tenu compte de la vue en contre-plongée qu'auraient les pèlerins en arrivant sous les marches qui donnent accès à la basilique :

«Parfaite l'abside avec ses chapelles rayonnantes, ce motif si heureux des églises romanes; très satisfaisantes, les façades latérales. Mais la façade principale? Nous l'osons dire manquée [...] Regardez d'en bas cette église sur son piédestal [...] Il vous semble ne voir qu'une grande chapelle. La coupole,

66. Joseph-Louis Duc, «Rapport sur le jugement du concours de l'église du Sacré-Cour », Souvenir du Concours de l'église du Sacré-Cour, op. cit., p. 15-16.

67. Julien Guadet, "L'église du Sacré-Cour ", La Revue de l'Art ancien et moderne, op. cit., p. 116-117.

68. Raboisson, «Exposition des projets de l'église du Sacré-Cœur ", Le Monde, 29 juillet 1874.

69. Claudius Lavergne, "Concours pour la construction de l'église du Sacré-Cour à Montmartre", L'Univers, $\mathrm{n}^{\circ} 2512$, dimanche 19 juillet 1874, p. 1-2.

70. "Programme d'un concours pour la construction de l'église du Sacré-Cœur de Montmartre", Le Moniteur des architectes, op. cit., p. 115. 
pivot de la composition, s'amoindrit dans la perspective, et comme premier plan, il n'y a qu'un porche [...] la façade n'est pas en proportion de l'édifice ${ }^{71}$.

Hubert Rohault de Fleury critiquait aussi chez Abadie son église "trop écrasée ", son dôme, "heureuse imitation de Saint Front [qui] ne se découvrirait pas de la place Saint-Pierre », et le campanile qui "serait tout à fait masqué derrière l'abside " ${ }^{72}$. Ce n'était pas l'avis de certains critiques pour qui l'église, phare de la chrétienté, devait être vue de loin :

"Quelques artistes se sont beaucoup préoccupés de l'effet que produirait l'église du pied de la butte; c'est là une grave erreur. Ce n'est pas du pied de la butte ou du marché de Montmartre que l'église doit être vue, mais de tous les points de Paris et de vingt lieux au loin ${ }^{73}$.

Par dessus tout, Paul Abadie fut critiqué pour " ce congrès de coupoles [...] remarquables surtout par leur étrangeté et leur caractère exotique » :

" [Il n'est pas étonnant après Saint-Front de Périgueux] qu'Abadie ait eu la pensée de renouveler la migration byzantine en essayant de l'implanter et de l'acclimater au centre de la France, en plein Paris [...] Passe encore s'il s'agissait du culte de saint Jean Chrysostôme ou d'un saint oriental quelconque ; mais pour le Sacré-Cœur, non, c'est une discordance de style et d'appropriation [...] ni les archéologues, ni la commission du Vœu national, ni le sens populaire ne sauraient concevoir que l'on pense à déloger les moulins à vent de Montmartre pour le plaisir de faire régner à leur place les coupoles de Trébisonde ${ }^{74}$.

L'abbé Carle y voyait surtout la main de l'Institut :

"Le concours a abouti, on le sait, hélas! au triomphe d'une école. Nous voulions un splendide temple, on va nous faire une mosquée, une manière d'église russe [...] Nous voulions un édifice qui traduisît l'élan de tout un peuple repentant et suppliant, on nous offre et l'on accepte pour nous un pastiche des plus malheureux, des plus mesquins; un dessin digne [...] non d'être réalisé sur le mont des Martyrs, mais digne tout au plus d'être reproduit sur des tabatières [...] Il y a là [dans la commission] cinq membres

71. Julien Guadet, "L'église du Sacré-Cœur », La Revue de l'Art ancien et moderne, op. cit., p. 114.

72. Hubert Rohault de Fleury, Historique de la Basilique du Sacré-Caeur, pièces et documents, op. cit., p. 208.

73. "Concours d'architecture pour la construction de l'église du Sacré-Cœur, à Paris-Montmartre", Le Bâtiment, journal des travaux publics et particuliers, $\mathrm{n}^{\circ}$ 50, dimanche 26 juillet 1874, p. 1.

74. Claudius Lavergne, "Concours pour la construction de l'église du Sacré-Cour à Montmartre", L'Univers, op. cit., p. 1-2. 
de l'Institut; à chacun sa coupole. C'est correct; qui donc oserait s'en plaindre? ${ }^{75}$.

Car cette " croisade contre la mosquée Abadie " ${ }^{76}$ cachait à peine la guerre larvée que se faisaient l'École des Beaux-Arts et l'école dite "archéologique » ${ }^{77}$. Il était écrit dans cet article au titre rageur que "ces messieurs" (les membres du comité du Vœu national) "n'osaient pas lutter contre la fameuse École des Beaux-Arts ». Sur les douze membres de la commission chargée de juger les projets, huit provenaient de l'École des Beaux-Arts et de celle des Ponts et chaussées, c'est-à-dire de " deux corps ennemis de l'architecture essentiellement nationale, du style gothique, qui ne veulent entendre parler que des styles des Grecs, des Romains et des Musulmans $"{ }^{78}$. L'obligation de signer les plans ${ }^{79}$ pour les exposer à l'École des Beaux-Arts avait éloigné les "archéologues et architectes de l'école gothique ». Dix ans avaient passé depuis la révolte de 1863 , et pourtant les querelles n'étaient pas éteintes. Les mêmes taxaient le dôme de Pascal de "gros dôme unique de la plus grande lourdeur " ${ }^{80}$. Le style byzantin était aussi critiqué car "favorisant le séjour des eaux du ciel sur les édifices et en compromettant la durée, dans nos pays exposés aux fréquentes pluies " ${ }^{81}$. Et le fait qu'Abadie se soit inspiré de l'appareil bicolore à assises alternées $^{82}$ de la cathédrale de Marseille ne plaidait pas en sa faveur : "Parce qu'à Marseille le regrettable Espérandieu a abusé de cet alternance dans ses appareils, sous un ciel pourtant plein d'éclat, sous les rayons d'un soleil presque oriental, pensezvous obtenir mieux que lui sous les brumes du nord de la France?». De plus, ce style n'assurait pas les bonnes conditions d'acoustique "qu'une église doit rechercher avant tout ${ }^{83}$. En somme le projet d'Abadie n'était pas rationaliste: "Nous avions cru jusqu'ici qu'il y a une carte archéologique imposée par la nature, le soleil, la configuration des lieux, la latitude et même les matériaux " ${ }^{84}$. Alors que "tout, dans l'architecture ogivale, dit le Dictionnaire raisonné, prend sa place et

75. L'église du Sacré-Cour à Montmartre sera-t-elle de notre style national ou sera-t-elle d'un style étranger?, par un comité d'archéologues, op. cit., p. 26.

76. Annotation d'un des numéros de la Décentralisation de Lyon du 14 juin 1875, envoyé par un "prêtre très influent " à l'un des secrétaires du comité du Vœu national. Ibid., p. 11.

77. L'école diocésaine. C'est pourquoi celle-ci fut très surprise de constater qu'Adolphe Napoléon Didron (1806-1867), le père des Annales archéologiques, se rangeait sous la bannière Abadie, dans un article du Monde du 30 juin 1875. Ibid., p. 19.

78. Ibid., p. 12.

79. Au lieu de les caractériser par une citation, ce qui assurait l'anonymat.

80. L'église du Sacré-Coeur à Montmartre sera-t-elle de notre style national ou sera-t-elle d'un style étranger?, par un comité d'archéologues, op. cit., p. 14.

81. Ibid., p. 47.

82. Voir les projets d'Abadie pour le concours, coupe transversale et perspective intérieure dans Claude Laroche, "Anatomie d'une chimère. Genèse et fortune du projet d'Abadie ", Paul Abadie, architecte. 1812-1894, op. cit., p. 235.

83. L'église du Sacré-Cour à Montmartre sera-t-elle de notre style national ou sera-t-elle d'un style étranger?, par un comité d'archéologues, op. cit., p. 48.

84. Ibid., p. 50. 
conserve sa qualité ${ }^{85}$. Les choses étaient pourtant plus nuancées en ce qui concerne Abadie, qui, bien que diocésain de la première heure après avoir œuvré pour la commission des monuments historiques, n'avait pourtant jamais critiqué publiquement l'École des Beaux-Arts dont il était issu ${ }^{86}$.

On va voir ressurgir, à l'occasion de la construction du Sacré-Cour, un autre des grands débats qui animèrent le milieu architectural du XIX ${ }^{\mathrm{e}}$ siècle, celui opposant les architectes et les archéologues : Julien Guadet se situe dans la lignée de Pascal, ses prises de position sur l'archéologie sont les mêmes, et, surtout, dans la lignée de Charles Garnier :

"C'est précisément en tant qu'il permet de mesurer le caractère relatif des réponses, et à ce titre seulement, que le savoir archéologique est utile, fécond et nécessaire [...] À part cela, enseigner l'archéologie est nuisible, privilégier le gothique, comme prétendent le faire les disciples de Viollet-le-Duc, est absurde : "L’archéologie, voilà l'ennemi !" énonce Guadet en reprenant les propos de son maître Garnier " 87 .

Charles Garnier était « le partisan de la création éclectique contre celui [Violletle-Duc] qu'il tenait, à tort, pour l'inconditionnel de l'archéologie " ${ }^{88}$, et l'on sait que Paul Abadie était un disciple fervent de Viollet-le-Duc. Julien Guadet voyait en Paul Abadie un "homme du passé ":

"Comme tout autre peut-être à sa place, il a dû reproduire plutôt que composer, son œuvre est une restauration plutôt qu'une création [...] Si ce fut une faiblesse - et nous le croyons - elle lui fut commune avec son époque. Et pourtant, combien n'est-elle pas insultante pour nous, artistes du XIX siècle, cette passion néfaste de l'archéologie qui nous crie: hors du passé, point de salut! ${ }^{89}$

et, comparant l'Opéra de Charles Garnier et le Sacré-Cœur de Paul Abadie :

"Chez l'un tous les affranchissements du passé, toute la compréhension du présent, tous les élancements vers l'avenir. Chez l'autre, la dévotion au passé, la méconnaissance du présent, la mainmise sur l'avenir par le passé [...] L'art religieux n'est encore aujourd'hui conçu que comme un art archéologique ${ }^{90}$.

85. Ibid., p. 58.

86. Claude Laroche, "Abadie et le rationalisme néo-médiéval ", Paul Abadie, architecte. 1812-1894, op. cit., p. 335-346.

87. Jean-Michel Leniaud, Les Bâtisseurs d'avenir. Portraits d'architectes. $X I X^{e}-X X^{e}$ siècle, Paris, Fayard, 1998, p. 124.

88. Ibid., p. 111.

89. Julien Guadet, «L'église du Sacré-Cour », La Revue de l'Art ancien et moderne, op. cit., p. 119.

90. Ibid., p. 108. 
Claude Laroche a montré depuis ${ }^{91}$ que le travail d'Abadie s'inspirait au moins autant du travail fait par lui précédemment à partir d'un modèle (ici, Saint-Front de Périgueux) que du modèle lui-même, ce qu'il appelle en reprenant une expression du chanoine J. Roux, les "filiations au second degré " 92 . Selon Jean-Michel Leniaud, "le Sacré-Cœur est en quelque sorte à l'architecture religieuse ce que l'Opéra est à l'architecture civile : l'expression la plus achevée de la synthèse ${ }^{93}$. Paul Abadie avait créé un type, celui de l'église de pèlerinage ${ }^{94}$. Et la souscription populaire ouverte au printemps 1871, pour en assurer le financement, connut un formidable succès ${ }^{95}$. Le projet de Pascal ne fut pas choisi, mais tout le monde loua sa beauté et le talent de son auteur :

"On sent que les brillantes études classiques faites par M. Pascal, lors de son séjour en Italie comme pensionnaire de l'Académie de France à Rome, ont donné à tout son projet des qualités de force et d'élégance qui ne se trouvent réunies à un aussi haut degré que dans les monuments antiques de la Ville Éternelle " ${ }^{96}$.

91. Claude Laroche, "L'architecte et son modèle : le lent et difficile dépassement de l'archéologie ", Paul Abadie, architecte 1812-1894, op. cit., p. 327-333.

92. J. Roux, "La basilique du Sacré-Cœur de Montmartre et Saint-Front de Périgueux ", La semaine religieuse du diocèse de Périgueux et Sarlat, $\mathrm{n}^{\text {os }} 45$ et 48, 8 et 9 novembre 1919, cité dans Claude Laroche, "Anatomie d'une chimère. Genèse et fortune du projet d'Abadie ", Paul Abadie, architecte. 1812-1894, op. cit., p. 238 et note 27, p. 249.

93. Jean-Michel Leniaud, "Du néo-classique au béton ", Ces églises du XIXe siècle, C. Bouchon, C. Brisac, N.-J. Chaline, J.-M. Leniaud, Amiens, Encrage éd., 1993, p. 37-80.

94. «Montmartre sera désormais le modèle de l'église de pèlerinage pour près de cinquante ans : la basilique du Rosaire à Lourdes, construite par Léopold Hardy, de 1883 à 1885, la basilique de Saint-Martin de Tours, de Victor Laloux (1887-1924), Notre-Dame de Brebières à Albert (18961901) d'Edmond Duthoit, et, pour finir, la basilique de Lisieux érigée en 1929 par Louis-Marie Cordonnier". Ibid., p. 67.

95. La Semaine Religieuse de Paris, vol. $\mathrm{n}^{\circ}$ XLII, $\mathrm{n}^{\circ} 1070,11$ juillet 1874 , p. $46-47$ (souscripteurs)-48 ; $\mathrm{n}^{\circ} 1071,18$ juillet 1874 , p. $75-76$ (souscripteurs) ; $\mathrm{n}^{\circ} 1072,25$ juillet 1874 , p. 112-114; $\mathrm{n}^{\circ} 1073$, $1^{\text {er }}$ août 1874, p. $147 ; n^{\circ} 1081,26$ septembre 1874, p. 396 (souscripteurs); no 1082, 3 octobre 1874, p. 432-434; n 1084,17 octobre 1874, p. 496 (souscripteurs)-497-502. L'opération rapporte 1200000 fr. en moins de dix ans, 40000000 fr. en quarante ans à raison d'un million par an. On estime à dix millions le nombre des souscripteurs dans une France de trente-cinq millions d'habitants. Jean-Michel Leniaud, "Coûts, fonds publics et souscriptions ", Ces églises du XIX siècle, op. cit., p. 128.

96. Charles Lucas, Un projet de décoration du Panthéon et le concours de l'église du Sacré-Cour à Montmartre, op. cit., p. 35. 
